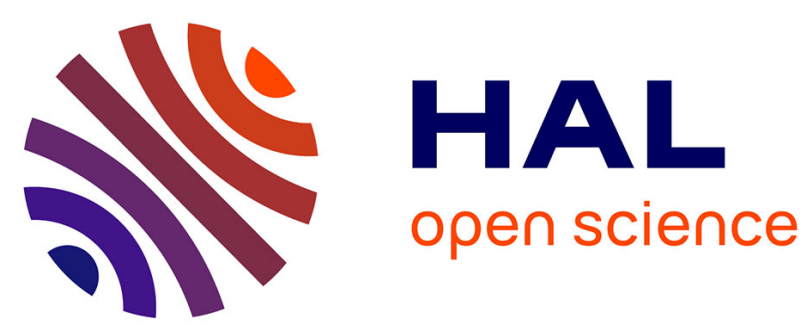

\title{
Fault Tolerant Control with Additive Compensation for Faults in an Automotive Damper
}

\author{
Juan C. Tudon-Martinez, Sébastien Varrier, Ruben Morales Menendez, \\ Ricardo Ramirez Mendoza, Damien Koenig, John Jairo Martinez Molina, \\ Olivier Sename
}

\section{To cite this version:}

Juan C. Tudon-Martinez, Sébastien Varrier, Ruben Morales Menendez, Ricardo Ramirez Mendoza, Damien Koenig, et al.. Fault Tolerant Control with Additive Compensation for Faults in an Automotive Damper. ICNSC 2013 - IEEE International Conference on Networking Sensing and Control, Apr 2013, Evry, France. hal-00937240

\section{HAL Id: hal-00937240 https://hal.science/hal-00937240}

Submitted on 28 Jan 2014

HAL is a multi-disciplinary open access archive for the deposit and dissemination of scientific research documents, whether they are published or not. The documents may come from teaching and research institutions in France or abroad, or from public or private research centers.
L'archive ouverte pluridisciplinaire HAL, est destinée au dépôt et à la diffusion de documents scientifiques de niveau recherche, publiés ou non, émanant des établissements d'enseignement et de recherche français ou étrangers, des laboratoires publics ou privés. 


\title{
Fault Tolerant Control with Additive Compensation for Faults in an Automotive Damper *
}

\author{
Juan C. Tudón-Martínez*, Sébastien Varrier ${ }^{\dagger}$, Ruben Morales-Menendez*, \\ Ricardo Ramirez-Mendoza*, Damien Koenig ${ }^{\dagger}$, John-Jairo Martinez ${ }^{\dagger}$ and Olivier Sename ${ }^{\dagger}$ \\ *Tecnológico de Monterrey, Av. Garza Sada 2501, 64849 Monterrey NL, México \\ Email: jc.tudon.phd.mty@itesm.mx \\ †Gipsa-Lab, 11 rue des mathématiques, 38402 Grenoble, France \\ Email: sebastien.varrier@gipsa-lab.fr
}

\begin{abstract}
A novel Fault-Tolerant Controller is proposed for an automotive suspension system based on a Quarter of Vehicle $(\mathrm{QoV})$ model. The design is divided in a robust Linear Parameter-Varying controller used to isolate vibrations from external disturbances and in a compensation mechanism used to accommodate actuator faults. The compensation mechanism is based on a robust fault detection and estimation scheme that reconstructs a fault on the semi-active damper; this information is used to reduce the failure effect into the vertical dynamics to achieve good control performances. Validations have been made over a $Q o V$ model in CarSim ${ }^{\mathrm{TM}}$. Results show the effectiveness of the faulttolerant semi-active damper versus an uncontrolled damper; the improvement is $\mathbf{5 0 . 4 \%}$ in comfort and $\mathbf{4 2 . 4 \%}$ in road holding, by avoiding biases in the damper deflection.
\end{abstract}

\section{INTRODUCTION}

Because advanced technological processes increase constantly their complexity, the control community has been developing novel techniques of modeling and control with fault-tolerance to improve the process reliability. A FaultTolerant Controller (FTC) is designed to maintain the desired performance of the system when occur process failures; there are two major groups: passive (when the fault tolerance is designed off-line) and active (based on an automatic control reconfiguration mechanism).

The importance of using an active system is to design a Fault Detection and Isolation (FDI) strategy. The FDI module estimates the fault on-line, which signal is used into the controller synthesis in order to accommodate the malfunction; in contrast, a passive version can result very conservative when different fault scenarios exist.

A reconfiguration mechanism of compensation is one of the different ways to accommodate the fault [1]; for the fault estimation, FDI modules based on analytical redundancy are the most accepted frameworks, in particular parity space approaches for Linear-Time Invariant (LTI) systems.

During the last years, the modeling of Linear ParameterVarying $(L P V)$ systems has gained importance as a solution to include the non-linear dynamics into the control law. Recently, $L P V$ based techniques have been extended to FTC approaches. For instance, in [2] it is proposed an FTC for polytopic $L P V$ systems by considering multiple failures.

\footnotetext{
* This work was partially supported by the Mexican PCP project $03 / 10$ and the French national project INOVE ANR 2010 BLAN 0308.
}

Particularly in automotive control applications, some researches on FTC are designed for active suspension systems: in [3] an FTC based on sliding mode observers is proposed, while in [4] a fault tolerant $L P V$ control is designed to guarantee road holding and roll stability; both FTC approaches are passive with good robustness. For semi-active suspension systems, a control strategy under different faulty schemes in a Quarter of Vehicle $(Q o V)$ model is proposed in [5], an on-line parametric estimation is used to create a fault signature by using parity relations; however, the fault is not estimated.

This paper proposes an active FTC for a semi-active suspension system, by analyzing a $Q o V$ model that includes an experimental Magneto-Rheological $(M R)$ damper model. The FTC structure is composed by a robust $L P V$ controller and a reconfiguration mechanism based on a robust fault detector. Based on $\mathcal{H}_{\infty}$ control theory, the $L P V$ controller is robust to road disturbances and oriented to comfort and road holding; while, the proposed FDI approach is an adaptation of the classical parity-space theory for the considered uncertain system, i.e. with un-modeled dynamics. The estimated fault obtained by the FDI module is used in a reconfiguration mechanism to compensate the fault effect in the vertical dynamics of the vehicle. Simulation results in $\mathrm{CarSim}^{\mathrm{TM}}$ shows the effectiveness of the proposed approach.

TABLE I. DEFINITION OF VARIABLES.

\begin{tabular}{|c|c|}
\hline Variable & Description \\
\hline$\rho_{1}$ & Varying parameter to represent hysteresis of the damper \\
$\rho_{1}^{*}$ & Varying parameter to represent hysteresis of the damper \\
$\rho_{2}^{*}$ & by using a filter in the control input \\
$a_{i}$ & Varying parameter to represent saturation of actuation \\
$b_{i}$ & Pre-yield viscous damping coefficients in $M R$ damper \\
$f_{c}$ & Post-yield viscous damping coefficients in $M R$ damper \\
$F_{M R}$ & Dynamic yield force in the $M R$ damper model \\
$F_{o}$ & $M R$ damper force \\
$I, I_{0}$ & Additive actuator fault \\
$I_{L P V}$ & Electric current, Mean of $I$ \\
$I_{F T C}$ & Electric current derived from the $L P V$ controller \\
$I_{F D I}$ & Electric current derived from the $F T C$ \\
$\mathcal{K}_{L P V}$ & Electric current derived from the $F D I$ \\
$k_{s}, k_{t}$ & Polytopic $L P V$ controller structure \\
$m_{s}, m_{u s}$ & Spring \& wheel stiffness coefficient \\
$r, \bar{r}$ & Sprung \& unsprung mass in the $Q o V$ \\
$z_{d e f}$ & Parity space residual \\
$\dot{z}_{d e f}$ & Damper piston position \\
$z_{r}$ & Damper piston velocity \\
$z_{s}, z_{u s}$ & Road profile \\
$\dot{z}_{s}, \dot{z}_{u s}$ & Vertical position of $m_{s}, m_{u s}$ \\
$\ddot{z}_{s}, \ddot{z}_{u s}$ & Vertical velocity of $m_{s}, m_{u s}$ \\
\hline
\end{tabular}


The paper is organized as follows: the problem statement is described in the next section. Sections III and IV describe the methodology of fault detection and estimation and the design of the FTC, respectively. Section V presents the simulation results. Finally, conclusions are presented in section VI. All variables are defined in Table I.

\section{PROBLEM STATEMENT}

A classical $Q o V$ model of a pick-up truck is used as testbed. An experimental $M R$ damper model represents the semiactive suspension between the sprung and unsprung masses, given by:

$$
\begin{aligned}
m_{s} \ddot{z}_{s} & =-k_{s}\left(z_{s}-z_{u s}\right)-F_{M R} \\
m_{u s} \ddot{z}_{u s} & =k_{s}\left(z_{s}-z_{u s}\right)-k_{t}\left(z_{u s}-z_{r}\right)+F_{M R}
\end{aligned}
$$

The semi-active damping force used for this approach is an extension of [6], but with inclusion of the manipulation signal (electric current). Its non-linear dynamics can be modeled by the damping force $F_{M R}$ as:

$$
F_{M R}(t)=I f_{c} \rho_{1}(t)+b_{1} \dot{z}_{d e f}(t)+b_{2} z_{d e f}(t)+F_{o}(t)
$$

where the non-linear part $\rho_{1}(t)$ is given by:

$$
\rho_{1}(t)=\tanh \left(a_{1} \dot{z}_{d e f}+a_{2} z_{d e f}\right)
$$

By using (1) and (2), a state-space representation of the QoV model can be obtained as:

$$
\begin{aligned}
& \underbrace{\left[\begin{array}{c}
\ddot{z}_{s} \\
\dot{z}_{s} \\
\ddot{z}_{u s} \\
\dot{z}_{u s}
\end{array}\right]}_{\dot{x}}=\underbrace{\left[\begin{array}{cccc}
-\frac{b_{1}}{m_{s}} & -\frac{c_{1}}{m_{s}} & \frac{b_{1}}{m_{s}} & \frac{c_{1}}{m_{s}} \\
1 & 0 & 0 & 0 \\
\frac{b_{1}}{m_{u s}} & \frac{c_{1}}{m_{u s}} & -\frac{b_{1}}{m_{u s}} & -\frac{c_{1}+k_{t}}{m_{u s}} \\
0 & 0 & 1 & 0
\end{array}\right]}_{A} \underbrace{\left[\begin{array}{c}
\dot{z}_{s} \\
z_{s} \\
\dot{z}_{u s} \\
z_{u s}
\end{array}\right]}_{x} \\
& +\underbrace{\left[\begin{array}{c}
-\frac{f_{c} \rho_{1}}{m_{s}} \\
0 \\
\frac{f_{c} \rho_{1}}{m_{u s}} \\
0
\end{array}\right]}_{B_{I}} I+\underbrace{\left[\begin{array}{c}
-\frac{1}{m_{s}} \\
0 \\
\frac{1}{m_{u s}} \\
0
\end{array}\right]}_{B_{F}} F_{o}+\underbrace{\left[\begin{array}{c}
0 \\
0 \\
\frac{k_{t}}{m_{u s}} \\
0
\end{array}\right]}_{B_{r}} z_{r} \\
& \underbrace{\left[\begin{array}{c}
\ddot{z}_{s} \\
\ddot{z}_{u s} \\
z_{d e f}
\end{array}\right]}_{y}=\underbrace{\left[\begin{array}{cccc}
-\frac{b_{1}}{m_{s}} & -\frac{c_{1}}{m_{s}} & \frac{b_{1}}{m_{s}} & \frac{c_{1}}{m_{s}} \\
\frac{b_{1}}{m_{u s}} & \frac{c_{1}}{m_{u s}} & -\frac{b_{1}}{m_{u s}} & -\frac{c_{1}+k_{t}}{m_{u s}} \\
0 & 1 & 0 & -1
\end{array}\right]}_{C}\left[\begin{array}{c}
\dot{z}_{s} \\
z_{s} \\
\dot{z}_{u s} \\
z_{u s}
\end{array}\right] \\
& +\underbrace{\left[\begin{array}{c}
-\frac{f_{c} \rho_{1}}{m_{s}} \\
\frac{f_{c} \rho_{1}}{m_{u s}} \\
0
\end{array}\right]}_{D_{I}} I+\underbrace{\left[\begin{array}{c}
-\frac{1}{m_{s}} \\
\frac{1}{m_{u s}} \\
0
\end{array}\right]}_{D_{F}} F_{o}+\underbrace{\left[\begin{array}{c}
0 \\
\frac{k_{t}}{m_{u s}} \\
0
\end{array}\right]}_{D_{r}} z_{r}
\end{aligned}
$$

\section{A. Problem definition}

Design an active FTC for the semi-active suspension system of a $Q o V$ model capable to mitigate the effects of an additive fault in the $M R$ damper. The FDI module aims to compensate the malfunction of the $M R$ damper by adding a compensatory signal to the $L P V$ controller. Both approaches ( $L P V$ controller and FDI module) must be designed to be robust to road disturbances and model uncertainties, and include the nonlinearities of the semi-active damper into its design, such that:

$$
\begin{aligned}
& \dot{x}=\mathcal{A}(\rho) \cdot x+\mathcal{B} \cdot\left[I_{F T C} F_{o} z_{r}\right]^{T} \\
& y=\mathcal{C}(\rho) \cdot x+\mathcal{D} \cdot\left[I_{F T C} F_{o} z_{r}\right]^{T} \\
& I_{F T C}=\mathcal{K}_{L P V}(\rho) \cdot x-I_{F D I}\left(\hat{F}_{o}\right)
\end{aligned}
$$

with $\mathcal{K}_{L P V}(\rho)=\sum_{i=1}^{N} \xi_{i}(\rho) \mathcal{K}_{i}$ by appropriately choosing the gains $\mathcal{K}_{i}, i=1, \ldots, N$ such that the closed-loop system (4) be asymptotically stable in all parameter variations, and $I_{F D I}$ depends on the estimated fault based on a nonlinear relation force - electric current.

\section{FAULT DETECTION AND ESTIMATION}

The fault detection scheme is based on the parity-space approach, because of its applicability to actuator faults in nonlinear systems, speed of detection, isolability property, robustness and computational complexity [1]. The aim is to synthesize a residual which is sensitive to the fault $F_{o}$ and insensitive to bounded exogenous inputs, e.g. the road profile or load transfers on the vehicle.

The outputs $y(t)$ are expressed along the horizon $s$ of the time derivatives as:

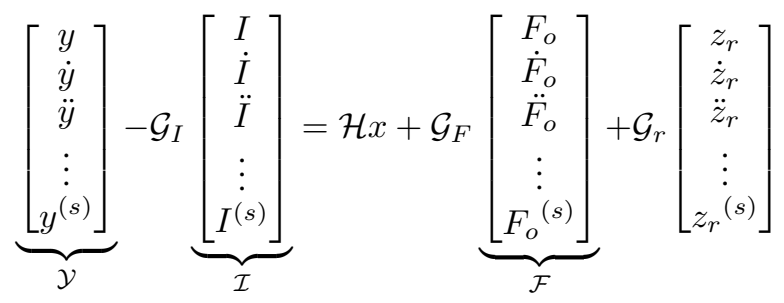

$$
\begin{aligned}
& \text { where } \mathcal{G}_{x}=\left[\begin{array}{cccccc}
D_{x} & 0 & \ldots & \ldots & \ldots & 0 \\
C B_{x} & D_{x} & 0 & \ldots & \ldots & 0 \\
C A B_{x} & C B_{x} & \ddots & \ddots & \ddots & \vdots \\
C A^{2} B_{x} & C A B_{x} & \ddots & \ddots & \ddots & \vdots \\
\vdots & \ddots & \ddots & \ddots & \ddots & 0 \\
C A^{s-2} B_{x} & C A^{s-3} B_{x} & \cdots & C A B_{x} & C B_{x} & D_{x}
\end{array}\right] \\
& \text { and } \mathcal{H}=\left[\begin{array}{c}
C \\
C A \\
C A^{2} \\
\vdots \\
C A^{s-1}
\end{array}\right] \text { ensures the observability condition. }
\end{aligned}
$$

To fulfill the requirement of sensitivity to the fault $F_{o}$ and insensitivity to road disturbances, the parity-matrix $\mathcal{W}$ is:

$$
\mathcal{W} \cdot\left[\begin{array}{lll}
\mathcal{H} & \mathcal{G}_{r}
\end{array}\right]=0
$$

Equation (6) guarantees the perfect decoupling of residuals from the states $x$ of the system and from the road disturbances $z_{r}$. The expression of the residuals is:

$$
r(t)=\mathcal{W}\left(\mathcal{Y}-\mathcal{G}_{I} \mathcal{I}\right)=\mathcal{W} \mathcal{G}_{F} F_{o}(t)
$$

where residuals $r(t)$ are perfectly sensitive to $F_{o}(t)$. By computing the null-space matrix $\mathcal{W}$ it will be possible to detect any additive fault in the damper without considering the road disturbances or the manipulation variable. 
It is well known that the $Q o V$ model does not include other non-linear dynamics of a real vehicle, e.g. mechanical joints, stabiliser bars, etc. Thus, the model-based residuals need to add special robustness, mainly because most of this un-modeled dynamics of the full vehicle affects in (3).

The expression of the residual (7) can be modified as :

$$
r(t)=\mathcal{W G}_{F} F_{o}(t)+\mathcal{W} \mathcal{G}_{\delta} \delta(t)
$$

where $\delta(t)$ represents exogenous unknown inputs (e.g. load transfers), and $\mathcal{G}_{\delta}$ its distribution on the system.

Perfect decoupling between $r(t)$ and those unknown inputs $\delta(t)$ is quite difficult because $\delta$ can be large; it is preferred an optimization approach [7]. In this study, the idea is to find a matrix $\mathcal{W}_{2}$ such that the new residual $\bar{r}(t)$ is:

$$
\bar{r}(t) \triangleq \mathcal{W}_{2} r(t)
$$

which is sensitive to the fault $F_{o}(t)$ and insensitive to $\delta(t)$; in terms of an optimization problem $\mathcal{P}$, this is:

$$
\mathcal{P}:\left\{\begin{array}{cc}
\max _{\mathcal{W}_{2}} & \left\|\mathcal{W}_{2} \mathcal{W} \mathcal{G}_{F}\right\|^{2} \\
\min _{\mathcal{W}_{2}} & \left\|\mathcal{W}_{2} \mathcal{W} \mathcal{G}_{\delta}\right\|^{2}
\end{array}\right.
$$

which by developing leads to:

$$
\mathcal{P}:\left\{\begin{array}{cc}
\max _{\mathcal{W}_{2}} & \mathcal{W}_{2} \mathcal{W} \mathcal{G}_{F} \mathcal{G}_{F}{ }^{T} \mathcal{W}^{T} \mathcal{W}_{2}{ }^{T} \\
\min _{\mathcal{W}_{2}} & \mathcal{W}_{2} \mathcal{W} \mathcal{G}_{\delta} \mathcal{G}_{\delta}{ }^{T} \mathcal{W}^{T} \mathcal{W}_{2}{ }^{T}
\end{array}\right.
$$

This $\min / \max$ optimization is rewritten in a single optimization problem as:

$$
\mathcal{P}: \min _{\mathcal{W}_{2}} \frac{\mathcal{W}_{2} \Gamma_{1} \mathcal{W}_{2}^{T}}{\mathcal{W}_{2} \Gamma_{2} \mathcal{W}_{2}^{T}}
$$

where $\Gamma_{1}=\mathcal{W} \mathcal{G}_{\delta} \mathcal{G}_{\delta}{ }^{T} \mathcal{W}^{T}$ and $\Gamma_{2}=\mathcal{W} \mathcal{G}_{F} \mathcal{G}_{F}{ }^{T} \mathcal{W}^{T}$, whose known solution, as recalled in [7], is:

$$
\mathcal{P} \Leftrightarrow \mathcal{W}_{2}=\vartheta_{-}\left(\Gamma_{1}, \Gamma_{2}\right)
$$

where $\vartheta_{-}\left(\Gamma_{1}, \Gamma_{2}\right)$ stands for the generalized eigenvector of the pair $\left(\Gamma_{1}, \Gamma_{2}\right)$, associated to the lowest eigenvalue $\lambda_{-}$.

Thus, the residual robust to un-modeled dynamics, via the matrix $\mathcal{G}_{\delta}$, has the following structure :

$$
r(t)=\overline{\mathcal{W}} \mathcal{G}_{F} F_{o}(t)+\overline{\mathcal{W}} \mathcal{G}_{\delta} \delta(t)
$$

where $\overline{\mathcal{W}}=\mathcal{W}_{2} \mathcal{W}$.

By assuming a good decoupling face to the uncertainties, the residual (14) can be rewritten as:

$$
r(t) \simeq \overline{\mathcal{W}} \mathcal{G}_{F} F_{o}(t)=\overline{\mathcal{W}}\left[\mathcal{G}_{F}{ }^{l} \mid \mathcal{G}_{F}{ }^{r}\right]+\left[\begin{array}{c}
F_{o} \\
\dot{F}_{o} \\
\vdots \\
F_{o}(s)
\end{array}\right]
$$

such that the fault $F_{o}$ can be estimated by the expression:

$$
\hat{F}_{o}=\left[\overline{\mathcal{W}} \mathcal{G}_{F}{ }^{l}\right]^{+}\left(r(t)-\mathcal{G}_{F}{ }^{r}\left[\begin{array}{c}
\dot{\hat{F}}_{o} \\
\vdots \\
\hat{F}_{o}^{(s)}
\end{array}\right]\right)
$$

where, the operator $[\cdot]^{+}$stands for the Moore Penrose pseudo inverse.

\section{FTC DESIGN}

The FTC design is divided in: 1) a robust $L P V$ controller used to isolate the vibrations in the vehicle caused by road disturbances and, 2) a reconfiguration mechanism which uses the fault detector (FDI) to accommodate the fault, Fig. 1.

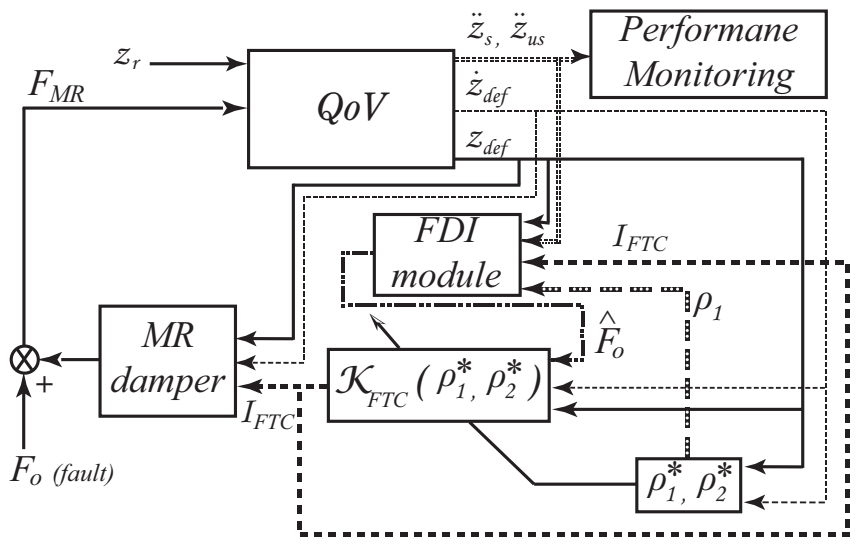

Fig. 1. Block diagram of the proposed FTC system.

Two varying parameters are used in the $L P V$ controller synthesis: $\rho_{1}^{*}$ includes the nonlinearities of the damper and $\rho_{2}^{*}$ is used to saturate the output controller. The FDI module estimates the fault $\hat{F}_{o}$, which is used to compute the required manipulation for compensating the fault effect in the actuator. The actuator fault is implemented in additive form.

Since the model contains the scheduling parameter into the control input matrix, it is required to add a filter to have a proper $L P V$ structure, [8]. Also, it is required to add a second scheduling parameter for ensuring the saturation of the control input. As an extension of [9], the new $L P V$ structure, robust to the actuator fault, is ruled by:

$$
\begin{aligned}
\dot{x}_{p} & =A_{p}\left(\rho_{1}^{*}, \rho_{2}^{*}\right) \cdot x_{p}+B_{p 1} \cdot u_{1}+B_{p 2} \cdot u_{2} \\
y & =C_{p} \cdot x_{p}+D_{p 1} \cdot u_{1}+D_{p 2} \cdot u_{2}
\end{aligned}
$$

where,

$$
\begin{aligned}
& x_{p}=\left[\begin{array}{c}
\dot{z}_{s} \\
z_{s} \\
\dot{z}_{u s} \\
z_{u s} \\
x_{f}
\end{array}\right], \quad A_{p}\left(\rho_{1}^{*}, \rho_{2}^{*}\right)=\left[\begin{array}{cc}
A+\rho_{2}^{*} B_{I 0} C_{I 0} & B_{I}\left(\rho_{1}^{*}\right) C_{f} \\
01 \times 4 & A_{f}
\end{array}\right] \\
& B_{p 1}=\left[\begin{array}{c}
0_{4 \times 1} \\
B_{f}
\end{array}\right], \quad B_{p 2}=\left[\begin{array}{cc}
B_{F} & B_{r} \\
0 & 0
\end{array}\right], \quad D_{p 1}=\left[0_{3 \times 1}\right] \\
& D_{p 2}=\left[\begin{array}{ll}
D_{F} & D_{r}
\end{array}\right], \quad C_{p}=\left[\begin{array}{ll}
C & D_{I}\left(\rho_{1}^{*}\right) C_{f}
\end{array}\right] \\
& u_{1}=I_{f} \quad u_{2}=\left[\begin{array}{c}
F_{o} \\
z_{r}
\end{array}\right] \quad B_{I 0}=\left[\begin{array}{cccc}
-\frac{I_{0}}{m_{s}} & 0 & \frac{I_{0}}{m_{s}} & 0
\end{array}\right]^{T}
\end{aligned}
$$

$$
I_{0}=\operatorname{mean}(I) \quad C_{I 0}=\left[\begin{array}{llll}
a_{1} & a_{2} & -a_{1} & -a_{2}
\end{array}\right]
$$

The scheduling parameters are defined as:

$$
\begin{array}{lll}
\rho_{1}^{*}=\rho_{1} \cdot \frac{\tanh \left(C_{f} x_{f} / I_{0}\right)}{C_{f} x_{f} / I_{0}} & \in[-1,1] \\
\rho_{2}^{*}=\frac{\rho_{1}}{C_{I 0} x} & \in[0,1]
\end{array}
$$


The filter design $\left(A_{f}, B_{f}, C_{f}, D_{f}\right)$ and $L P V$ modeling with constraints in the actuator can be reviewed in [8], [9].

The polytopic approach for $L P V$ control design bounds the nonlinearities of a closed-loop system into a finite dimension problem. A polytope of 4 LTI controllers is designed by using $\mathcal{H}_{\infty}$ control theory; the controller is quadratically stable for all trajectories of the varying parameters by using $L M I$ techniques, [10]. The weighting functions are designed to reduce the vertical acceleration of the sprung mass (comfort) and the displacement of the unsprung mass (road holding):

$$
W_{\ddot{z}_{s}}=\frac{K_{s}\left(s^{2}+2 \zeta_{1 s} \omega_{1 s} s+\omega_{1 s}{ }^{2}\right)}{s^{2}+2 \zeta_{2 s} \omega_{2 s} s+\omega_{2 s}{ }^{2}}, \quad W_{z_{u s}}=\frac{K_{u s}\left(s+\omega_{1 u s}\right)}{s+\omega_{2 u s}}
$$

Taking into account the control specifications, the generalized system $\mathcal{P}$ for the $L P V$ control synthesis is:

$$
\begin{aligned}
\dot{x}_{p} & =A_{p}\left(\rho_{1}^{*}, \rho_{2}^{*}\right) \cdot x_{p}+B_{p 1} \cdot u_{1}+B_{p 2} \cdot u_{2} \\
z & =C_{z}\left(\rho_{1}^{*}, \rho_{2}^{*}\right) \cdot x_{p} \cdot\left[\begin{array}{cc}
W_{\ddot{z}_{s}} & 0 \\
0 & W_{z_{u s}}
\end{array}\right] \\
y & =C_{p} \cdot x_{p}+D_{p 1} \cdot u_{1}+D_{p 2} \cdot u_{2}
\end{aligned}
$$

where $C_{z}\left(\rho_{1}^{*}, \rho_{2}^{*}\right)=\left[\begin{array}{ll}C_{z 1} & \rho_{1}^{*} D_{z 1} C_{f}\end{array}\right], D_{z 1}=\left[\begin{array}{c}-\frac{f c}{m_{s}} \\ 0\end{array}\right]$ and,

$$
C_{z 1}=\left[\begin{array}{cccc}
-\frac{b_{1}}{m_{s}} & -\frac{c_{1}}{m_{s}} & \frac{b_{1}}{m_{s}} & \frac{c_{1}}{m_{s}} \\
0 & 0 & 1 & 0
\end{array}\right]+\left[\begin{array}{c}
-\frac{\rho_{2}^{*} I_{0}}{m_{s}} C_{I 0} \\
0_{1 \times 4}
\end{array}\right]
$$

The reconfiguration of compensation uses the estimated fault $\hat{F}_{o}$ for computing the required electric current to correct the malfunction. This non-linear relation is given by the inverse dynamics of the $M R$ damper model, eq. (2), such that:

$$
I_{F D I}=I_{0} \cdot \tanh \left(\frac{\hat{F}_{o}-b_{10} \dot{z}_{d e f}-b_{20} z_{d e f}}{f_{0} \cdot \rho_{1}}\right) \in\left[-I_{0}, I_{0}\right]
$$

where, the tanh function bounds the non-linear relation $I-F_{M R}$ around the average of the electric current $\left(I_{0}\right)$, by considering $f c=f_{0}, b_{1}=b_{10}$ and $b_{2}=b_{20}$ from an experimental test at passive damping conditions.

Equation (20) represents the electric current required to reduce the fault propagation into the vehicle dynamics; because this fault is additive, $I_{F D I}$ must subtract the effect of the estimated fault $\hat{F}_{o}$, as a compensation, in the closed-loop of (3). Because the actuation over the damper is saturated, the magnitude of the fault affects directly the bandwidth of control. Thus, the fault-tolerant controller output is composed by the robust $L P V$ controller output $\left(I_{L P V}\right)$ and the compensation $\left(I_{F D I}\right)$ as:

$$
I_{F T C}=I_{L P V}-I_{F D I}
$$

such that $B_{F} \cdot F_{o}-B_{I} \cdot I_{F D I} \sim 0$ in the closed-loop system in order to eliminate the fault effects. The resulting closed-loop dynamics is given by:

$$
\dot{x}=A x+B_{I}\left(\mathcal{K}_{L P V} x-I_{F D I}\right)+B_{F} F_{o}+B_{r} z_{r}
$$

where, $\mathcal{K}_{L P V}$ is the polytopic $L P V$ controller obtained by the $L M I$ solution.

\section{RESULTS AND DISCUSSION}

The considered suspension system (test-bed) is obtained from a customized pick-up truck model by using a $K \& C$ test, which dynamics is generated via Carsim ${ }^{\mathrm{TM}}$. The analysis is carried on the front-left corner of the vehicle, whose QoV model parameters are: $m_{s}=470 \mathrm{Kg}, m_{u s}=110 \mathrm{Kg}$, $k_{s}=86,378 N . m^{-1}$ and $k_{t}=270,000 N . m^{-1}$. For damper modeling, experimental data were identified at two different electric current values from a standardized test (road elevation chirp), the model parameters are: $f c=600.95, a_{1}=37.85$, $a_{2}=22.15, b_{1}=2,830.86$ and $b_{2}=-7,897.21$.

The vehicle is moving straight forward at constant speed $v=30 \mathrm{~km} / \mathrm{h}$. The road profile is composed of two successive bumps of $0.1 \mathrm{~m}$ height at $t=0.6 \mathrm{~s}$ and $t=3.7 \mathrm{~s}$, which are considered as disturbances for the FDI module. The fault occurs between both bumps, at $t=1 \mathrm{~s}$. The fault is a negative force appearing on the damper: $F_{0}=-4000 \mathrm{~N}$, i.e. around $40 \%$ of the operation span in the rebound effect. This kind of fault could be caused by a bias of reference into the inner controller of the semi-active damper, i.e. it is a fault in the actuation system.

The measurements of the $Q o V$ suspension system, using $\operatorname{CarSim}^{\mathrm{TM}}$ as software-in-the-loop in Matlab/Simulink ${ }^{\mathrm{TM}}$, are used for fault estimation according to the methodology presented in section III. Figure 2 shows that the fault estimation is not perfect, but it converges to the implemented fault. Note that the FDI module is insensitive to the bumps (road disturbances). This information can be directly compared with a threshold to warn the driver about the faulty damper.

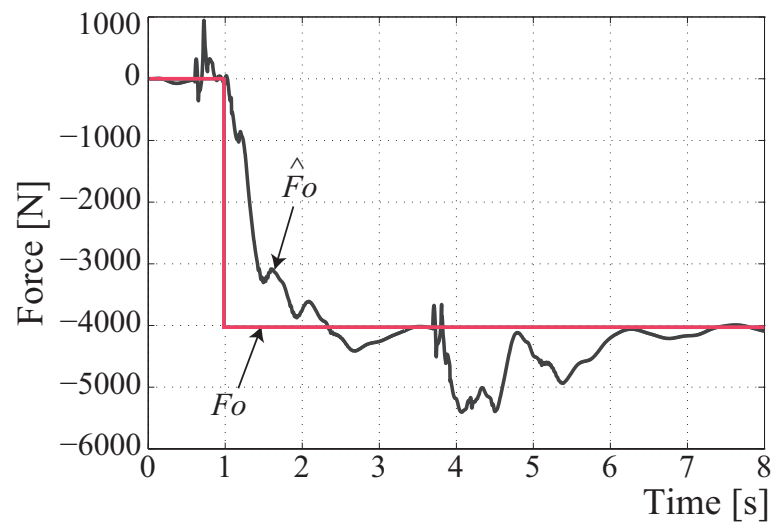

Fig. 2. Fault estimation.

Moreover, a suspension control system of the vehicle could take into account this fault estimation in order to maintain the desired performance of comfort and/or road holding.

Plots in Fig. 3 compare the performance of an uncontrolled shock absorber respect to the controlled one with faulttolerance features (Fault-Tolerant Semi-Active: FTSA damper) based on the control design proposed in section IV.

Note in Fig. 3 that before $t=1 s$ (fault free case) the FTSA and uncontrolled system behave identically. When the fault occurs, $t>1 \mathrm{~s}$, the sprung mass acceleration is reduced when the FTSA damper is used, Fig. 3a; the oscillations due to the bumps are more attenuated. The comfort of the passengers is increased in contrast to the uncontrolled damper that loses damping capability when the fault occurs. By computing the 

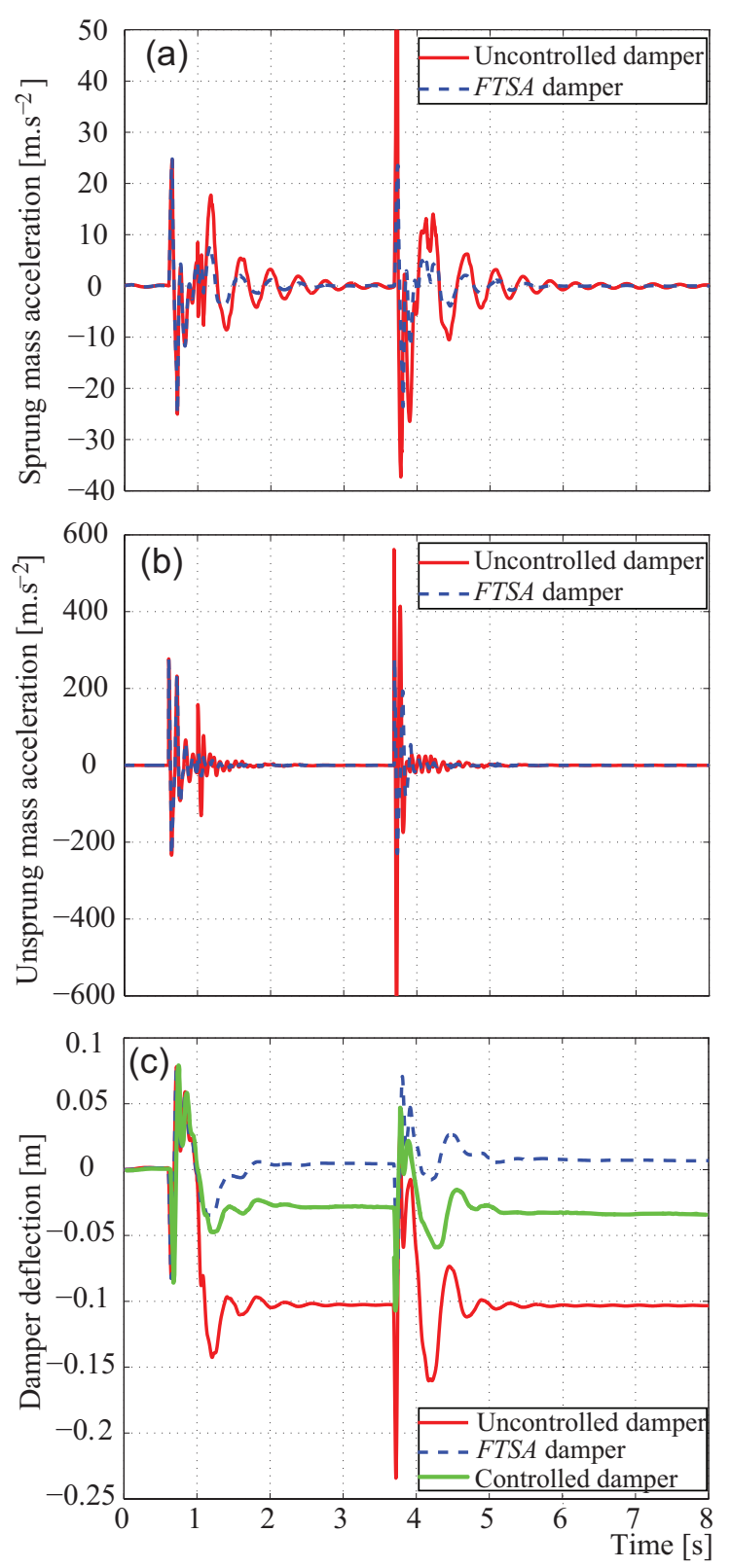

Fig. 3. Comparison between an uncontrolled and FTSA damper.

Root Mean Square $(R M S)$ value of the $\ddot{z}_{s}$, the FTSA damper presents $3.31 \mathrm{~m} / \mathrm{s}^{2}$ versus to $6.68 \mathrm{~m} / \mathrm{s}^{2}$ of the uncontrolled damper, i.e. an improvement of $50.4 \%$ of comfort.

At the same time, Fig. 3b shows that the unsprung mass acceleration is also better due to the fault accommodation. These lower accelerations cause lower $z_{u s}$ and consequently lower deflection between the tire and road, i.e. an improvement of $42.4 \%$ in road holding (using the $R M S$ of $\ddot{z}_{u s}$ ).

Figure $3 \mathrm{c}$ shows the deflection between the masses, i.e. it is a monitor of the damper stroke. The robust fault information plus the robust control compensates the effect of the fault. During the second bump $(t=3.7 s)$, the magnitude of the deflection is more attenuated. The uncontrolled damper is clearly affected by the fault, its stroke will remain roughly 10 $\mathrm{cm}$ lower than its normal position. This bias into the stroke does not only affect the motion in the sprung and unsprung mass; it also could affects dramatically the life-time of the damper, i.e. this bias could cause that the damper piston arrives until the jounce-rebound stops.

Also Fig. 3c presents a comparison between the FTSA damper and a controlled damper which only uses the robust $L P V$ feature without the reconfiguration mechanism. Although the $L P V$ controller is designed to reduce the vibrations in the sprung and unsprung masses when external disturbances (e.g. the road profile) and faults occur, results show that this control approach is not good enough; in this case, the fault causes a bias in the damper stroke around $4 \mathrm{~cm}$ lower than its normal position. Inclusion of all possible faulty schemes is a drawback of passive FTC approaches. On the other hand, the proposed active FTC compensates the lack of effectiveness of the damper and permits to behave like a safe actuator. Early results show promising real implementations; the sampling frequency of the electronic control unit must ensure at least the response time of the $M R$ damper ( $25 \mathrm{~ms})$.

\section{CONCLUSIONS}

An effective fault tolerant semi-active damper is achieved by synthesizing separately a robust fault estimator and controller. The idea was validated with a front-left $Q o V$ model using $\mathrm{CarSim}^{\mathrm{TM}}$. Once the fault is detected, its estimation is used to compensate the effect; however, the magnitude of the fault could limit the bandwidth of control. The robustness of the controller/fault estimator shows good performances even if the model is not perfect. Simulation results show that the proposed fault-tolerant controller improves comfort $50.4 \%$ and road holding $42.4 \%$ and maintains the nominal position of the stroke when an additive fault occurs in the damper.

\section{REFERENCES}

[1] Y. Zhang and J. Jiang, "Bibliographical Review on Reconfigurable Fault-tolerant Control Systems", Annual Reviews in Control, vol. 32, 2008, pp: 229-252.

[2] M. Rodrigues, D. Theilliol, S. Aberkane and D. Sauter, "Fault Tolerant Control Design for Polytopic LPV Systems", Int. J. AMCS, vol. 17(1), 2007, pp: 27-37.

[3] A. Chamseddine and H. Noura, "Control and Sensor Fault Tolerance of Vehicle Active Suspension", IEEE Trans. on Control Systems Tech., 16(3), 2008, pp: 416-433.

[4] P. Gáspár, Z. Szabó and J. Bokor, "LPV Design of Fault-tolerant Control for Road Vehicles", SysTol'10, France, 2010, pp: 807-812.

[5] D. Fischer and R. Isermann, "Mechatronic Semi-active and Active Vehicle Suspensions", Control Eng Practice, vol. 12, 2004, pp: 13531367.

[6] S. Guo, S. Yang and C. Pan, "Dynamical Modeling of Magnetorheological Damper Behaviors", J. of Intell. Mater., Syst. and Struct., vol. 17, 2006, pp: 3-14

[7] S. Varrier, D. Koenig and J.J. Martinez, "Robust Fault Detection for Vehicle Lateral Dynamics", 51 ${ }^{\text {st }}$ IEEE Conf. on Decision and Control, Hawai USA, 2012, pp: 4366-4371.

[8] C. Poussot-Vassal, O. Sename, L. Dugard, P. Gáspár, Z. Szabó and J. Bokor, "A New Semi-active Suspension Control Strategy through LPV Technique", Control Eng. Practice, vol. 16, 2008, pp: 1519-1534.

[9] A.L. Do, O. Sename and L. Dugard, "An LPV Control Approach for Semi-Active Suspension Control with Actuator Constraints", American Control Conf., USA, 2010, pp: 4653-4658.

[10] C. Scherer, P. Gahinet and M. Chilali, "Multiobjective Output-feedback Control Via LMI Optimization", IEEE Trans. on Automatic Control, 42(7), 1997, pp: 896-911. 